This is an Open Access article, distributed under the terms of the Creative Commons Attribution licence (http://creativecommons.org/licenses/by/4.0/), which permits unrestricted re-use, distribution, and reproduction in any medium, provided the original work is properly cited.

\title{
Signatures of quantum effects on radiation reaction in laser-electron-beam collisions
}

\author{
C. P. Ridgers ${ }^{1} \dagger$, T. G. Blackburn ${ }^{2}$, D. Del Sorbo ${ }^{1}$, L. E. Bradley ${ }^{1}$, \\ C. Slade-Lowther ${ }^{1}$, C. D. Baird ${ }^{1}$, S. P. D. Mangles ${ }^{3}$, P. McKenna ${ }^{4}$, \\ M. Marklund ${ }^{2}$, C. D. Murphy ${ }^{1}$ and A. G. R. Thomas ${ }^{5,6,7}$ \\ ${ }^{1}$ York Plasma Institute, Department of Physics, University of York, Heslington, York YO10 5DD, UK \\ ${ }^{2}$ Department of Physics, Chalmers University of Technology, SE-41296 Gothenburg, Sweden \\ ${ }^{3}$ The John Adams Institute for Accelerator Science, Blackett Laboratory, Imperial College London, \\ South Kensington, London SW7 2BZ, UK \\ ${ }^{4}$ Department of Physics SUPA, University of Strathclyde, Glasgow G4 0NG, UK \\ ${ }^{5}$ Center for Ultrafast Optical Science, University of Michigan, Ann Arbor, MI 48109-2099, USA \\ ${ }^{6}$ Cockcroft Institute, Daresbury Laboratory, Warrington WA4 4AD, UK \\ ${ }^{7}$ Department of Physics, Lancaster University, Lancaster LA1 4YB, UK
}

(Received 28 May 2017; revised 4 August 2017; accepted 7 August 2017)

Two signatures of quantum effects on radiation reaction in the collision of a $\sim \mathrm{GeV}$ electron beam with a high intensity $\left(>3 \times 10^{20} \mathrm{~W} \mathrm{~cm}^{-2}\right)$ laser pulse have been considered. We show that the decrease in the average energy of the electron beam may be used to measure the Gaunt factor $g$ for synchrotron emission. We derive an equation for the evolution of the variance in the energy of the electron beam in the quantum regime, i.e. quantum efficiency parameter $\eta \ll 1$. We show that the evolution of the variance may be used as a direct measure of the quantum stochasticity of the radiation reaction and determine the parameter regime where this is observable. For example, stochastic emission results in a $25 \%$ increase in the standard deviation of the energy spectrum of a $\mathrm{GeV}$ electron beam, 1 fs after it collides with a laser pulse of intensity $10^{21} \mathrm{~W} \mathrm{~cm}^{-2}$. This effect should therefore be measurable using current high-intensity laser systems.

Key words: intense particle beams, plasma dynamics, plasma simulation

\section{Introduction}

Radiation reaction is the effective recoil force on an accelerating charged particle caused by the particle emitting electromagnetic radiation. This effect will play an important role in laser-matter interactions at the intensities set to be reached by next generation high-intensity laser facilities $\left(\gtrsim 10^{23} \mathrm{~W} \mathrm{~cm}^{-2}\right)$, where radiation reaction can lead to almost complete absorption of the laser pulse: Bashinov \& Kim (2013) (using a classical theory) and Zhang, Ridgers \& Thomas (2015) (including quantum corrections), have shown that radiation reaction gives an imaginary part in the

$\dagger$ Email address for correspondence: christopher.ridgers@york.ac.uk 
dispersion relation for waves in a plasma. At intensities $\gtrsim 10^{23} \mathrm{~W} \mathrm{~cm}^{-2}$, plasma electrons will become sufficiently energetic that in their individual rest frames the electric field $E_{\mathrm{RF}}$ approaches the critical field for quantum electrodynamics $E_{\text {crit }}=1.38 \times 10^{18} \mathrm{~V} \mathrm{~m}^{-1}$ (Heisenberg \& Euler 1936). In this case, the emission of radiation by the electrons must be described in the framework of strong-field quantum electrodynamics (QED), using the Furry (1951) picture. Specifically, when the quantum efficiency parameter $\eta=E_{\mathrm{RF}} / E_{\text {crit }} \gtrsim 0.1$ the radiation reaction force becomes stochastic (Duclous, Kirk \& Bell 2011) and electron's dynamics is no longer well approximated by deterministic motion along a classical worldline (Shen \& White 1972).

This quantum regime has been reached in experiments on the Super Proton Synchrotron at CERN in the interaction of $\sim 100 \mathrm{GeV}$ electrons with the strong fields of atoms in a crystal lattice, as described by Andersen et al. (2012), where the Gaunt factor for synchrotron emission was measured. The analogous process of nonlinear Compton scattering was studied experimentally at the Stanford Linear Accelerator (SLAC) in the interaction between an electron beam of energy $E=46.6 \mathrm{GeV}$ and a counter-propagating high intensity $\left(10^{18}-10^{19} \mathrm{~W} \mathrm{~cm}^{-2}\right)$ laser pulse, as reported by Bula et al. (1996) (positron generation was also observed in this experiment - see Burke et al. 1997). In this experiment the laser intensity was too low to access the very nonlinear regime of relevance to next generation laser-matter interactions, where $a_{0} \approx \sqrt{I \lambda^{2} / 10^{18} \mathrm{~W} \mathrm{~cm}-2 \mu \mathrm{m}^{2}} \gg 1(\lambda$ is the laser wavelength). This is now possible with current Petawatt laser systems, which can achieve focused intensities of $I>10^{21} \mathrm{~W} \mathrm{~cm}^{-2}$. In the interaction of an electron beam with energy $\mathcal{E}$ with a counter-propagating laser pulse of intensity $I, \eta$ can be estimated as $\eta \sim 0.1(\mathcal{E} / 500 \mathrm{MeV}) \sqrt{I / 10^{21} \mathrm{~W} \mathrm{~cm}^{-2}}$. The quantum, nonlinear regime of Compton scattering and the resultant radiation reaction can therefore be studied by accelerating the electrons to energies greater than $500 \mathrm{MeV}$. Laser wakefield acceleration (Tajima \& Dawson 1979) is a technique that can generate monoenergetic, well collimated and ultra-relativistic electron beams (Faure et al. 2004; Geddes et al. 2004; Mangles et al. 2004). Recent experiments have now demonstrated energies approaching $5 \mathrm{GeV}$ (Leemans et al. 2014). Laser wakefield accelerators are ideal for studying electron-beam collisions with the tightly focused lasers required for studies of nonlinear Compton scattering due to the inherent synchronicity of the generated electron beam and the laser which allows precise overlap in space and time. Therefore, all-optical equivalents of the SLAC experiment are possible using PW lasers (Sokolov et al. 2010; Bulanov et al. 2012; Thomas et al. 2012; Neitz \& Di Piazza 2013; Blackburn et al. 2014; Vranic et al. 2014; Blackburn 2015). Nonlinear Compton scattering at $a_{0} \simeq 2$ (but not radiation reaction) was recently observed in such a set-up by Sarri et al. (2014). Devising ways in which quantum effects on radiation reaction can be distinguished is therefore timely, as has been considered by Di Piazza, Hatsagortsyan \& Keitel (2010), Neitz \& Di Piazza (2013), Blackburn et al. (2014), Vranic et al. (2015), Wang, Yan \& Zepf (2015) and Harvey et al. (2017).

To simplify the treatment of quantum radiation reaction, we use the quasiclassical approach described by Bă̌er \& Katkov (1968). Here, we assume that the electromagnetic fields may be split into two types depending on their frequency scale. Fields varying on the scale of the laser frequency are treated as classical background fields. The photons emitted by the electrons on acceleration by these background fields, i.e. those responsible for the radiation reaction force, are treated in the framework of strong-field QED. These photons are of much higher energy (typically $h v \gtrsim \mathrm{MeV})$ than the laser photons $(h v \sim \mathrm{eV})$. Two further simplifying 
approximations are made (see Kirk, Bell \& Arka 2009). By making the quasi-static approximation we assume that the formation length of the hard photons is much smaller than the scale over which the background fields vary and thus the background fields may thus be treated as constant over the space-time interval during which the emission occurs. This approximation is valid for $a_{0} \gg 1$, which is the case in high-intensity laser-matter interactions (Di Piazza et al. 2010 has shown that $a_{0} \gtrsim 10$ is sufficient). By making the weak-field approximation, we assume that the emission rate of photons depends entirely on $\eta$ and not the field invariants $\mathcal{F}=\left(E^{2}-c^{2} B^{2}\right) / E_{\text {crit }}^{2}$ and $\mathcal{G}=c \boldsymbol{E} \cdot \boldsymbol{B} / E_{\text {crit }}^{2}$. This is valid if these invariants are much smaller than $\eta$. For next generation laser-matter interactions $E, c B \lesssim 10^{-3} E_{\text {crit }}$, so this approximation is also reasonable. The weak-field approximation allows us to assume that the rate of photon emission (and the energy spectrum of the emitted photons) is well described by the well-known rate in an equivalent set of constant fields as given in Ritus (1985) (for constant crossed electric and magnetic fields) and Erber (1966) (for a constant magnetic field). The accuracy of this quasi-classical approach has recently been demonstrated by comparison to full QED calculations for the electron energies and laser intensities considered here by Dinu et al. (2016).

Using this quasi-classical model (making the quasi-static and weak-field approximations), it is possible to include the quantum radiation reaction force in a kinetic equation describing the evolution of the electron distribution, as given by Shen \& White (1972), Sokolov et al. (2010), Elkina et al. (2011), Neitz \& Di Piazza (2013) and Ridgers et al. (2014). Although this equation has been solved numerically using a Monte Carlo algorithm (see Duclous et al. 2011; Elkina et al. 2011; Ridgers et al. 2014; Gonoskov et al. 2015) it has not been solved analytically for even the simplest configuration of electromagnetic fields (for example a uniform, static magnetic field as in Shen \& White 1972). On the other hand, the electron equation of motion containing a classical model of radiation reaction, using the prescription of Landau \& Lifshitz (Landau \& Lifshitz (1987) - shown to be consistent with the classical limit of strong-field QED by Krivitskii \& Tsytovich (1991), Ilderton \& Torgrimsson (2013)), has been solved analytically in several cases for example: for electron motion in a rotating electric field (by Bell \& Kirk 2008) and a plane electromagnetic wave (by DiPiazza 2008). A modified classical model, where the radiated power is reduced by the Gaunt factor, has been used to derive the dispersion relation for an electromagnetic wave moving through a plasma where the electrons experience significant radiation reaction by Zhang et al. (2015) (and the equivalent classical result by Bashinov \& Kim 2013). The kinetic equation can be used to show that the modified classical model of radiation reaction is sufficient to describe the average energy loss of the electrons (Ridgers et al. 2014). In addition, the kinetic equation can give insight into which observables can be used to measure various aspects of quantum radiation reaction. Here we show that the measurements of the average energy loss can be used to measure the Gaunt factor associated with the emission and that the evolution of the variance of the electron energy distribution can be used to measure the degree of stochasticity of the emission. To do the latter, we derive an equation of motion for the variance, which extends the results of Vranic et al. (2015) to arbitrary $\eta$.

\section{Radiation reaction models}

In this section we describe the radiation reaction models considered here: (i) classical - using the ultra-relativistic form of the Landau and Lifshitz prescription; (ii) modified classical - as the classical model but including a function describing the reduction in the power radiated due to quantum effects, the Gaunt factor $g$ 
(Baier, Katkov \& Strakhovenko 1991); (iii) stochastic - a probabilistic treatment of the emission consistent with the approximations made in the quantum emission model described above and in more detail by Ridgers et al. (2014). The stochastic model is the most physical as it includes both the important quantum effects (the Gaunt factor and quantum stochasticity).

Using the quasi-classical approach we may write the evolution of the electron distribution function, including the radiation reaction force, as

$$
\frac{\partial f}{\partial t}+\boldsymbol{v} \cdot \frac{\partial f}{\partial \boldsymbol{r}}-e(\boldsymbol{E}+\boldsymbol{v} \times \boldsymbol{B}) \cdot \frac{\partial f}{\partial \boldsymbol{p}}=\left(\frac{\partial f}{\partial t}\right)_{\mathrm{em}}^{X} .
$$

$f d^{3} \boldsymbol{x} d^{3} \boldsymbol{p}$ is the number of electrons at position $\boldsymbol{x}$ with momentum $\boldsymbol{p}$ (velocity $\boldsymbol{v}$ ). $\boldsymbol{E}$ and $\boldsymbol{B}$ are the low frequency classical background electromagnetic fields. $(\partial f / \partial t)_{\mathrm{em}}^{X}$ is an operator describing how recoil from photon emission affects the electron distribution function - we will refer to this as the emission operator. The superscript $X$ denotes which of the classical $(\mathrm{cl})$, modified classical $(\bmod c l)$ and stochastic $(s t)$ models is under consideration.

Note that we are neglecting pair production by the emitted gamma-ray photons in the background electromagnetic fields. This is reasonable in the moderately quantum regime described by Di Piazza et al. (2010), i.e. where $\eta \sim 0.1$.

\subsection{Classical and modified classical emission operators}

If the radiating electron is ultra-relativistic with $\gamma \gg 1$, we may assume that all photons are emitted in the direction of the electron's instantaneous velocity (Duclous et al. 2011). Using the Landau and Lifshitz prescription for radiation reaction (in the ultra-relativistic limit - Landau \& Lifshitz 1987) the classical and modified classical emission operators should describe radiation reaction forces of the form

$$
\boldsymbol{F}_{\mathrm{cl}}=-\frac{P_{\mathrm{cl}}}{c} \hat{\boldsymbol{p}} \quad \boldsymbol{F}_{\operatorname{modcl}}=-\frac{g P_{\mathrm{cl}}}{c} \hat{\boldsymbol{p}}
$$

respectively. Here $g(\eta)$ is the Gaunt factor for synchrotron emission, i.e. a function that gives the reduction in the radiated power $P_{\mathrm{cl}}$ due to quantum modifications to the synchrotron spectrum. $P_{\mathrm{cl}}$ is parameterised in terms of $\eta$ as

$$
P_{\mathrm{cl}}=\frac{2 \alpha_{f} c}{3 \hbar_{c}} m_{e} c^{2} \eta^{2}
$$

where $\alpha_{f}$ is the fine-structure constant, $t_{c}$ is the reduced Compton wavelength and $g(\eta)$ is defined as

$$
g(\eta)=\frac{\int_{0}^{\eta / 2} F(\eta, \chi) \mathrm{d} \chi}{\int_{0}^{\infty} F_{\mathrm{cl}}\left(\frac{4 \chi}{3 \eta^{2}}\right) \mathrm{d} \chi}=\frac{3 \sqrt{3}}{2 \pi \eta^{2}} \int_{0}^{\eta / 2} F(\eta, \chi) \mathrm{d} \chi .
$$

$F_{\mathrm{cl}}$ and $F$ are the classical and quantum synchrotron spectra respectively. For completeness their forms are given in appendix A. An accurate fit to this function is $g(\eta) \approx\left[1+4.8(1+\eta) \ln (1+1.7 \eta)+2.44 \eta^{2}\right]^{-2 / 3}$ (Baier et al. 1991).

The emission operators which yield radiation reaction forces as given in (2.2), as shown in $\S 3$, are

$$
\left(\frac{\partial f}{\partial t}\right)_{\mathrm{em}}^{\mathrm{cl}}=\frac{1}{p^{2}} \frac{\partial}{\partial p}\left(p^{2} \frac{P_{\mathrm{cl}}}{c} f\right) \quad\left(\frac{\partial f}{\partial t}\right)_{\mathrm{em}}^{\bmod c l}=\frac{1}{p^{2}} \frac{\partial}{\partial p}\left(p^{2} g \frac{P_{\mathrm{cl}}}{c} f\right) .
$$




\subsection{Stochastic emission operator}

The stochastic emission operator should consist of two terms: a term describing the movement of electrons out of a given region of phase space due to emission and a term describing electrons moving into the region under consideration by leaving regions of higher energy as they emit. Assuming the electrons are ultra-relativistic and so photon emission is in the direction of propagation of the electron, we may formulate this as

$$
\left(\frac{\partial f}{\partial t}\right)_{\mathrm{em}}^{\mathrm{st}}=-\lambda_{\gamma}(\eta) f+\frac{b}{2 m_{e} c} \int_{p}^{\infty} \mathrm{d} p^{\prime} \lambda_{\gamma}\left(\eta^{\prime}\right) \rho_{\chi}\left(\eta^{\prime}, \chi\right) \frac{p^{\prime 2}}{p^{2}} f\left(\boldsymbol{p}^{\prime}\right) .
$$

We define $\eta \equiv \gamma b$. For $\gamma \gg 1$, we may take $b=\left|\boldsymbol{E}_{\perp}+\boldsymbol{v} \times \boldsymbol{B}\right| / E_{s} . \chi=(h \nu b) /\left(2 m_{e} c^{2}\right)$ is the quantum efficiency parameter for an emitted photon (with energy $h v$ ). The explicit form of the photon emission rate $\lambda_{\gamma}$ and the probability $\rho_{\chi} \mathrm{d} \chi$ that an electron with energy parameterised by $\eta$ emits a gamma-ray photon with energy parameterised by $\chi$ are given in appendix A.

\section{Moment equations}

The average over the distribution function $f$ of a momentum dependent quantity $\psi(\boldsymbol{p})$ is defined as

$$
\langle\psi(\boldsymbol{p})\rangle \equiv \frac{1}{n_{e}} \int d^{3} \boldsymbol{p} \psi(\boldsymbol{p}) f(\boldsymbol{x}, \boldsymbol{p}, t),
$$

where $n_{e}$ is the electron number density.

\subsection{The temporal evolution of $\langle\boldsymbol{p}\rangle$}

The equation for the evolution of the expectation value of the momentum of the electron population $\langle\boldsymbol{p}\rangle$ has been derived previously by Elkina et al. (2011). The equation for the evolution of the average energy $\langle\gamma\rangle$ of the population has been derived by Ridgers et al. (2014):

$$
\left(\frac{\mathrm{d}\langle\boldsymbol{p}\rangle}{\mathrm{d} t}\right)_{s t}=-\frac{\left\langle g P_{\mathrm{cl}} \hat{\boldsymbol{p}}\right\rangle}{c} .
$$

In appendix $\mathrm{B}$ we show how this equation can be derived by taking the first moment of the stochastic emission operator in (2.6).

Taking the first moment of the classical and modified classical emission operators given in (2.5), as detailed in appendix B, yields

$$
\left(\frac{\mathrm{d}\langle\boldsymbol{p}\rangle}{\mathrm{d} t}\right)_{\mathrm{cl}}=-\frac{\left\langle P_{\mathrm{cl}} \hat{\boldsymbol{p}}\right\rangle}{c} \quad\left(\frac{\mathrm{d}\langle\boldsymbol{p}\rangle}{\mathrm{d} t}\right)_{\operatorname{modcl}}=-\frac{\left\langle g P_{\mathrm{cl}} \hat{\boldsymbol{p}}\right\rangle}{c} .
$$

\subsection{The temporal evolution of $\sigma^{2}$}

Following the derivation in appendix B we can obtain the following equation for the evolution of the variance $\sigma^{2}$ in the Lorentz factor $\gamma$ of the electron distribution:

$$
\left(\frac{\mathrm{d} \sigma^{2}}{\mathrm{~d} t}\right)_{s t}=-2 \frac{\left\langle\Delta \gamma g P_{\mathrm{cl}}\right\rangle}{m_{e} c^{2}}+\frac{\langle S\rangle}{m_{e}^{2} c^{4}} .
$$

$\sigma^{2}=\left\langle\gamma^{2}\right\rangle-\langle\gamma\rangle^{2}$ and $\Delta \gamma=\gamma-\langle\gamma\rangle$. The first term in (3.4), which we label $T_{-}$, always acts to reduce the variance. It arises because higher energy electrons radiate more 


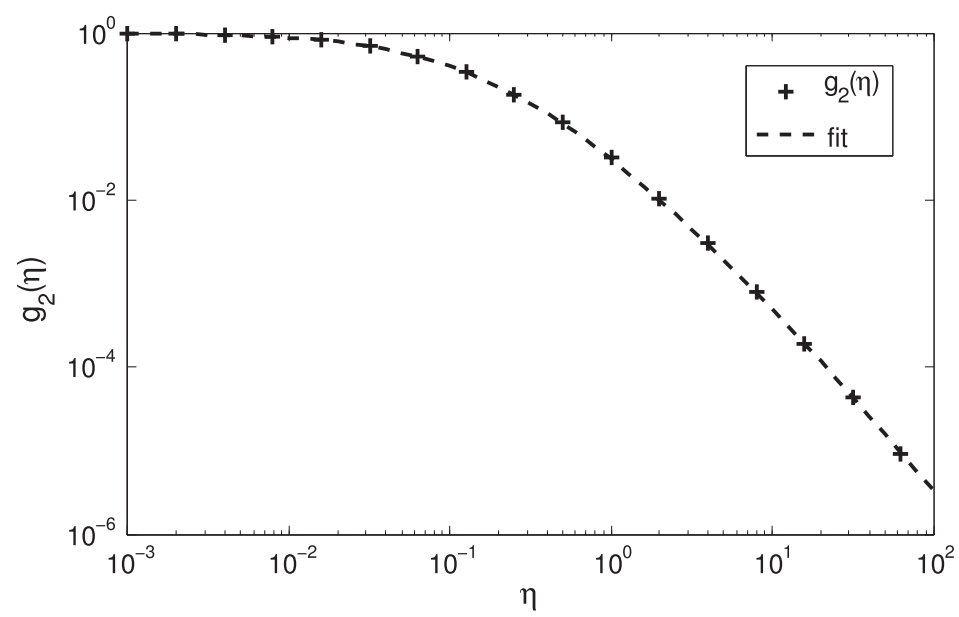

FIGURE 1. $g_{2}(\eta)$ (solid line) and the fit used here (dashed line).

energy than those at lower energy. This term can be written $T_{-}=\left(2 / m_{e} c^{2}\right)\left[\left\langle\Delta \gamma P_{\mathrm{cl}}\right\rangle-\right.$ $\left.\left\langle(1-g) \Delta \gamma P_{\mathrm{cl}}\right\rangle\right]$, where the first term is purely classical and the second shows that quantum effects reduce the rate of decrease of the variance by reducing the power radiated below the classical prediction $(g \leqslant 1)$. The second term in (3.4) $T_{+}$represents stochastic effects, is positive and so tends to increase the variance. The competition between these two terms determines whether the emission operator causes $\sigma^{2}(t)$ to increase or decrease.

The function $S(\eta)$ is given by

$$
S(\eta)=\frac{55 \alpha_{f} c}{24 \sqrt{3} \lambda_{c} b} m_{e}^{2} c^{4} \eta^{4} g_{2}(\eta) .
$$

$g_{2}(\eta)$, which is analogous to $g(\eta)$, is defined as

$$
g_{2}(\eta)=\frac{\int_{0}^{\eta / 2} \chi F(\eta, \chi) \mathrm{d} \chi}{\int_{0}^{\infty} \chi F_{\mathrm{cl}}\left(\frac{4 \chi}{3 \eta^{2}}\right) \mathrm{d} \chi}=\frac{144}{55 \pi \eta^{4}} \int_{0}^{\eta / 2} \chi F(\eta, \chi) \mathrm{d} \chi .
$$

As for $g$, it is useful to find an accurate fit to $g_{2}$. We find the following $g_{2}(\eta) \approx$ $\left[1+(1+4.528 \eta) \ln (1+12.29 \eta)+4.632 \eta^{2}\right]^{-7 / 6}$. This gives the correct limits for $\eta \ll 1$ and $\eta \gg 1\left(g_{2} \approx 1\right.$ and $g_{2} \approx 0.167 \eta^{-7 / 3}$ respectively). $g_{2}$, as a function of $\eta$, along with the fit are shown in figure 1.

We may also derive the corresponding expressions for $\mathrm{d} \sigma^{2} / \mathrm{d} t$ from the classical and modified classical emission operators in (2.5) (the derivation is given in appendix B).

$$
\left(\frac{\mathrm{d} \sigma^{2}}{\mathrm{~d} t}\right)_{\mathrm{cl}}=-2 \frac{\left\langle\Delta \gamma P_{\mathrm{cl}}\right\rangle}{m_{e} c^{2}} \quad\left(\frac{\mathrm{d} \sigma^{2}}{\mathrm{~d} t}\right)_{\operatorname{modcl}}=-2 \frac{\left\langle\Delta \gamma g P_{\mathrm{cl}}\right\rangle}{m_{e} c^{2}} .
$$

We now consider the specific case where a high energy electron beam with Gaussian energy distribution collides with a plane electromagnetic wave. In the limit 


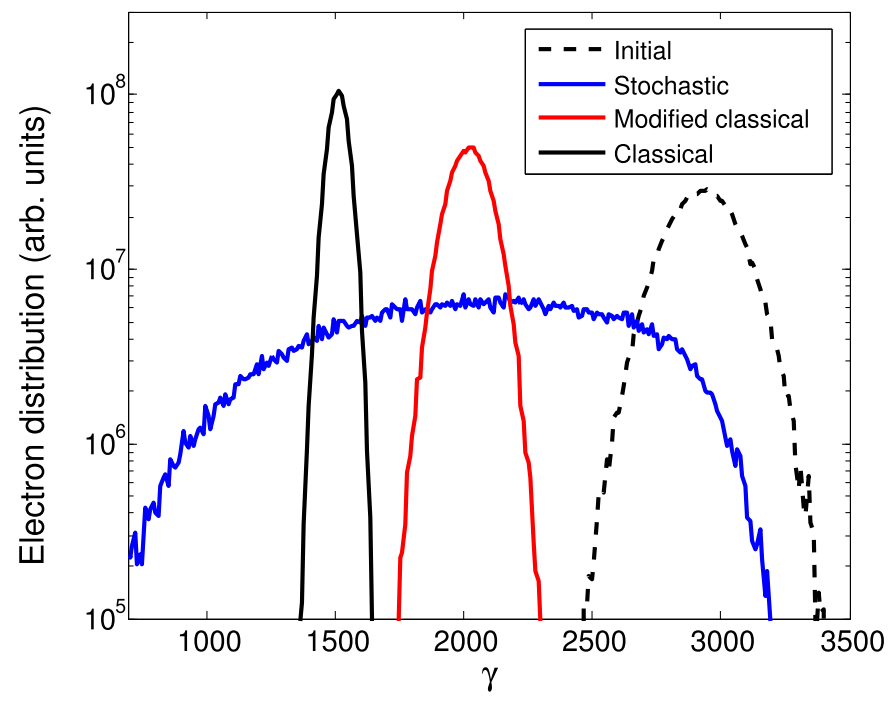

FIGURE 2. Electron energy distribution, 10.5 fs after collision of the electron bunch with the laser pulse, compared to initial distribution using the stochastic, modified classical and classical emission operators.

where $\eta \ll 1$ and the energy distribution is Gaussian with $\sigma \ll\langle\gamma\rangle$ (and assumed to be a Gaussian at all times), equation (3.4) reduces to

$$
\left(\frac{\mathrm{d} \sigma^{2}}{\mathrm{~d} t}\right)_{s t} \approx \frac{\alpha_{f} c b^{2}}{\lambda_{c}}\left(\frac{55 b}{24 \sqrt{3}}\langle\gamma\rangle^{4}-\frac{8}{3} \sigma^{2}\langle\gamma\rangle\right),
$$

which reproduces Vranic et al. (2015, equation (14)).

\section{Comparison to QED-PIC simulations}

To test the validity of the expression for the evolution of $\sigma^{2}$ given above we have simulated the interaction of an electron beam with a counter-propagating circularly polarised plane wave using the QED-Particle-in-Cell (PIC) code EPOCH (Arber et al. 2015). EPOCH includes the stochastic emission model using a Monte Carlo algorithm (described in detail by Ridgers et al. 2014). For this work we have extended the code to include the classical and modified classical emission operators by directly solving (2.2) using first-order Eulerian integration.

The simulation parameters were as follows. The laser pulse had peak intensity $10^{21} \mathrm{~W} \mathrm{~cm} \mathrm{~cm}^{-2}$, wavelength $1 \mu \mathrm{m}$ and a half-Gaussian temporal profile (rise time $1 \mathrm{fs}$ ). Four thousand grid cells were used to discretise a spatial domain extending from $-40 \mu \mathrm{m}$ to $40 \mu \mathrm{m}$ and $10^{5}$ macroparticles were used to represent an electron bunch consisting of $10^{9}$ electrons. The electron bunch had a Gaussian spatial profile, centred on $39.7 \mu \mathrm{m}$, with a full width at half maximum (FWHM) of $0.17 \mu \mathrm{m}$ and had initial distribution $f(\boldsymbol{x}, \boldsymbol{p}, t=0)=\left[n_{e}(\boldsymbol{x}) /(\sqrt{2 \pi} \sigma)\right] \delta\left(p_{y}\right) \delta\left(p_{z}\right) \exp \left[-\left(p_{x}+\gamma_{0} m_{e} c\right)^{2} /\left(2 \sigma^{2}\right)\right]$ where $\boldsymbol{p}=\left(p_{x}, p_{y}, p_{z}\right)$ is the momentum coordinate in phase space and $n_{e}$ the number density of electrons in the beam. $\gamma_{0}$ was the initial average energy of the bunch.

Figure 2 shows a comparison of the spatially integrated electron energy distribution using classical, modified classical and stochastic emission operators with the initial 

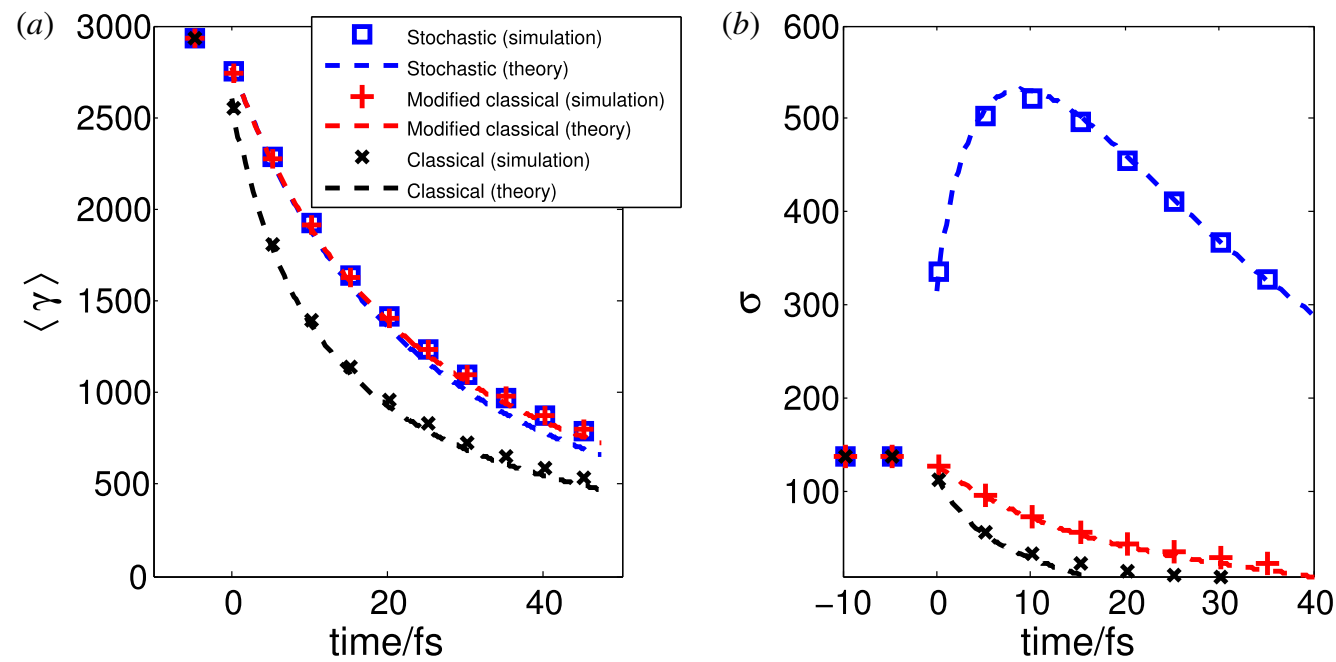

Figure 3. (a) Mean Lorentz factor versus time using the various emission models from simulation and as predicted by (3.2) and (3.3). (b) Standard deviation in Lorentz factor versus time from simulation and as predicted by (3.4) and (3.7).

spectrum $t=10.5 \mathrm{fs}$ after the collision. We see that the modified classical and classical emission operators both give a decrease in the variance of the electron distribution whereas the stochastic emission operator gives an increase in the variance. Figure 3 shows the temporal evolution of the mean Lorentz factor $\langle\gamma\rangle$ and the standard deviation of the Lorentz factor $\sigma$. The QED-PIC simulations demonstrate the validity of (3.2)-(3.7).

We saw earlier in (3.4) that the evolution of the variance is governed by the competition between $T_{-}$and $T_{+}$. To characterise which of these terms is dominant (in a similar way to Vranic et al. 2015), and thereby how stochastic quantum effects (prevalent when $T_{+}$dominates), may be measured in a colliding beams experiment, we derive an analytical expression their ratio $\xi$ :

$$
\xi=\frac{T_{+}}{T_{-}} \quad T_{+}=\frac{\langle S\rangle}{m_{e}^{2} c^{4}} \quad T_{-}=2 \frac{\left\langle\Delta \gamma g P_{\mathrm{cl}}\right\rangle}{m_{e} c^{2}} .
$$

Considering an electron bunch whose initial distribution is $f\left(\boldsymbol{x}, p_{x}, t=0\right)=$ $n_{e}(\boldsymbol{x}) /\left(2 W \gamma_{0} m_{e} c\right) \delta\left(p_{y}\right) \delta\left(p_{z}\right)$ for $\gamma_{0} m_{e} c(1-W)<\left|p_{x}\right|<\gamma_{0} m_{e} c(1+W)$ and assuming $g=g_{2}=1$, we obtain (as outlined in appendix C)

$$
\xi \approx\left(3.0+1.5 W^{-2}+0.3 W^{2}\right) \eta_{0},
$$

where $\xi$ is the ratio $T_{+} / T_{-}$when the electron bunch first collides with the laser pulse (i.e. before the distribution $f$ has evolved under the action of radiation reaction) and $\eta_{0}=\gamma_{0} b$. As the variance increases and the expectation value of the $\gamma$ decreases we expect $T_{-}$to eventually become dominant and so we would expect the variance to peak and then decrease after some time. This behaviour is clearly seen in the results from the simulation using the stochastic emission operator shown in figure 3 . Therefore, we define $T_{+}$as being important for $\xi>2$ initially in order to compensate for the increased importance of $T_{-}$at later times. In the case where the width of the 

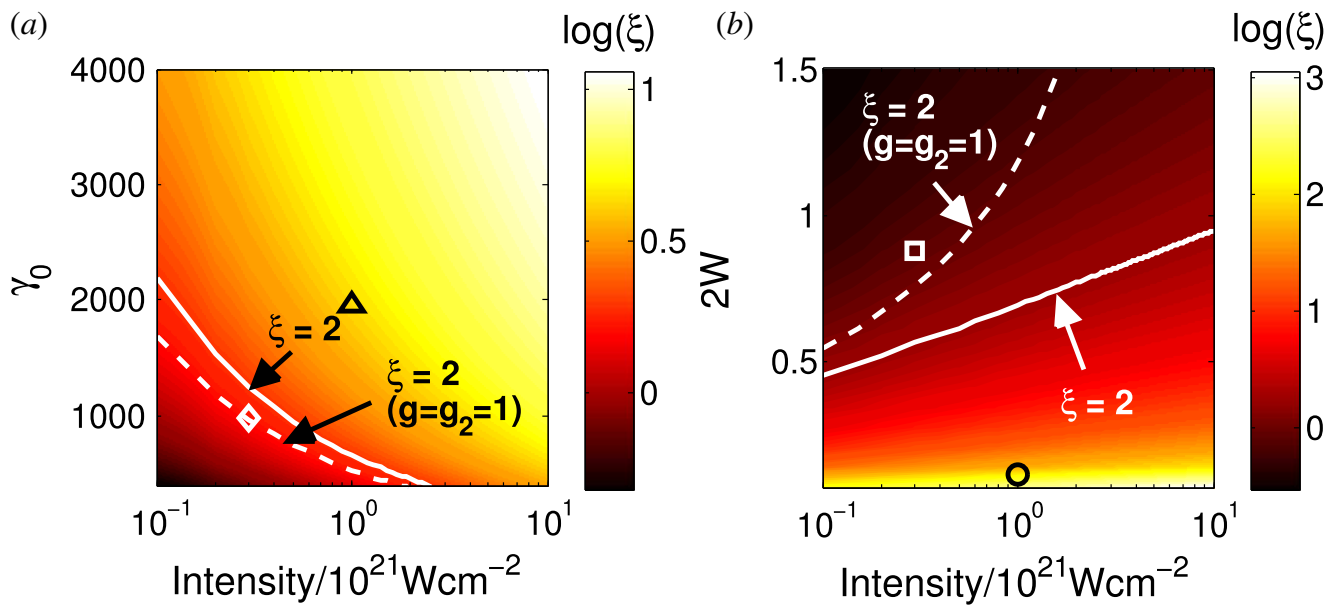

FIGURE 4. $\xi$ as a function of: laser intensity and average Lorentz factor of the electron bunch $(a)$; laser intensity and width of the electron energy distribution $(b)$. The solid white lines show $\xi=2$ and the dashed white lines show the prediction of where $\xi=2$ from (4.2).

$\begin{array}{lcccc}\text { Simulation } & I / 10^{21} \mathrm{~W} \mathrm{~cm}^{-2} & \gamma_{0} m_{e} c^{2} \mathrm{GeV}^{-1} & \text { FWHM GeV } & \text { Symbol } \\ 1 & 1.0 & 1.0 & 0.81 & \triangle \\ 2 & 0.3 & 0.5 & 0.21 & \diamond \\ 3 & 1.0 & 1.5 & 0.17 & \bigcirc \\ 4 & 0.3 & 1.5 & 1.3 & \square\end{array}$

TABLE 1. Simulation parameters used to investigate the dominance of $T_{+}$over $T_{-}$and vice versa

electron distribution is equal to the mean, $W=0.5$, equation (4.2) shows that $\eta_{0}>0.2$ is required for $\xi>2$. For a narrow electron distribution, $W \ll 1, \eta_{0}>1.3 W^{2}$ is required and so $T_{+}$can be important at lower $\eta_{0}$.

From (4.2) we see that $\xi$ depends on three variables: the average Lorentz factor of the electron bunch $\gamma_{0}$; the width of the electron energy distribution $W$ and the laser intensity $I$ (which determines $b$ ). Figure 4 shows $\xi$ (including $g$ and $g_{2}$ ) as a function of $I$ and $\gamma_{0}$ (for $W=0.2$ ) and $W$ and $I$ (for $\gamma_{0} m_{e} c^{2}=1.5 \mathrm{GeV}$ ). The prediction of $\xi=2$ from (4.2), i.e. making the assumption $g=g_{2}=1$, is shown to be reasonably accurate for $I \lesssim 10^{21} \mathrm{~W} \mathrm{~cm}^{-2}$.

To investigate whether the expression for $\xi$ in (4.2) predicts whether $T_{+}$or $T_{-}$ dominates the evolution of the variance we performed further EPOCH simulations of the interaction of an electron beam (again with initial distribution $f(\boldsymbol{x}, \boldsymbol{p}, t=0)=$ $\left.\left[n_{e} /(\sqrt{2 \pi} \sigma)\right] \delta\left(p_{y}\right) \delta\left(p_{z}\right) \exp \left[-\left(p_{x}+\gamma_{0} m_{e} c\right)^{2} /(2 \sigma)^{2}\right]\right)$ and a counter-propagating plane wave of intensity $I$. The following parameters were chosen:

We have shown where these simulations lie in the parameter space shown in figure 4 according to the symbols given in the table 1 and assuming $W=\sqrt{2} \sigma$. The time evolution of the change in the standard deviation of the electron energy distribution in these simulations is shown in figure 5. We see that only those simulations where (4.2) predicts that $T_{+}$is dominant show an increase in the variance. 


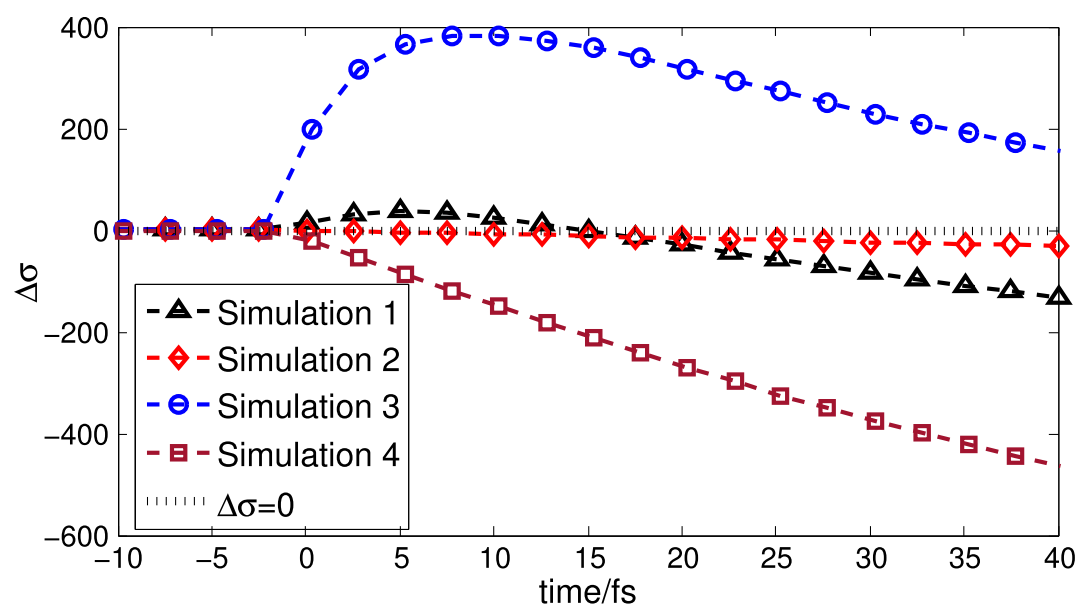

FIgURE 5. Temporal evolution of the change in standard deviation in the electron energy distribution in simulations $1-4$.

\section{Discussion}

The results of this investigation can be summarised as follows:

(i) $\langle\boldsymbol{p}\rangle$ evolves in the same way for the stochastic and modified classical emission operators and differently for the classical emission operator.

(ii) $\sigma^{2}$ evolves differently for all operators. In particular, the stochastic emission operator can result in an increase in $\sigma^{2}$ whereas the classical and modified classical operators can only cause a decrease in $\sigma^{2}$ (as seen by Vranic et al. (2015) for $\eta \ll 1)$.

Result (i) requires further explanation. Although we have shown that $(\mathrm{d}\langle\boldsymbol{p}\rangle / \mathrm{d} t)_{s t}$ and $(\mathrm{d}\langle\boldsymbol{p}\rangle / \mathrm{d} t)_{\bmod c l}$ evolve according to the same equation, it does not necessarily follow that the expectation values themselves are the same for these two emission models (as noted by Elkina et al. 2011). We have previously shown in Ridgers et al. (2014) that, in fact, the expectation values of the energy using these two models do agree to a high degree of accuracy and this was shown again for the parameters considered here in figure 3 . We would expect this in the classical limit where $\eta \ll 1$. In this case $T_{-}$in (3.4) dominates (from (4.2) we see that $\xi \propto \eta_{0}$ ) and rapidly reduces the variance of the electron bunch; the electron distribution in both the modified classical and stochastic models approaches a delta function $\delta(\boldsymbol{p}-\langle\boldsymbol{p}\rangle)$. The time evolution of $\langle\boldsymbol{p}\rangle$ depends on $\left\langle P_{\mathrm{cl}}\right\rangle\left(g \approx 1\right.$ in the classical limit) which is equal to $(\langle\eta\rangle) P_{\mathrm{cl}}(\langle\eta\rangle)$ for both the stochastic and modified classical models when $f$ is narrow in momentum space. However, in the simulation whose results are shown in figure $3 \eta>0.1$. From figure 2 we see that in this case the electron energy distribution is very different when the stochastic emission operator is used compared to when the modified classical emission operator is used and in the former case is certainly not narrow. Despite this the evolution of $\langle\boldsymbol{p}\rangle$ is the same due to the functional form of $g P_{\mathrm{cl}}$. When $\eta \gg 1$, $g P_{\mathrm{cl}} \propto \eta^{2 / 3}$. This almost linear dependence on $\eta$ means that the difference in the evolution of $\langle\boldsymbol{p}\rangle$ between the models should be small. Finally we note that, as shown in figure 3, $\langle\boldsymbol{p}\rangle$ predicted by the classical emission model differs markedly from that predicted by the modified classical and stochastic models due to the neglect of the Gaunt factor $g$ in the classical model.

$\mathrm{d} \sigma^{2} / \mathrm{d} t$ is always negative for both the classical and modified classical emission operators. Physically, this is because electrons at higher energy radiate more energy 
than those at lower energy, causing a decrease in the width of the energy distribution. The classical operator predicts a more rapid decrease than the modified classical operator due to the assumption that $g=1$ and the consequent overestimate of the scaling of the power radiated by the electrons with increasing $\eta$. For the stochastic emission operator $\mathrm{d} \sigma^{2} / \mathrm{d} t$ can be either positive or negative and so $\sigma^{2}$ can increase or decrease. The evolution of $\sigma^{2}$ is determined by the balance between $T_{+}$(which causes $\sigma^{2}$ to increase due the probabilistic nature of the emission) and $T_{-}$(which, as just described, causes $\sigma^{2}$ to decrease as higher energy electrons radiate more energy). We have shown (as did Vranic et al. 2015) that which of these terms dominates depends on the width of the energy distribution and $\eta$. For large width $T_{-}$increases in importance as it depends on $\Delta \gamma=\gamma-\langle\gamma\rangle$. For high $\eta T_{+}$becomes more important due to its scaling with $\eta^{4}$ compared to at most $\eta^{3}$ for $T_{-}$(assuming $\Delta \gamma \sim \gamma$ ). In (4.2) we have provided a formula for the determination of which term is dominant.

The first of these results, i.e. that the evolution of the expectation value is the same for the modified classical and stochastic (but not classical) models, is useful in two ways. Firstly it shows that measuring the expectation value of an electron bunch after interaction with a high-intensity laser pulse can give information about one quantum effect: the reduction of the total power emitted as expressed by $g$. It cannot, however, give information about the probabilistic nature of the emission. Secondly, this result suggests that the modified classical model of radiation reaction is sufficient for the calculation of laser absorption in high-intensity laser-plasma interactions (Brady et al. 2012; Zhang et al. 2015). Laser absorption in this context depends on the average energy loss by the electrons (and positrons) in the plasma due to radiation reaction. The second result, i.e. the evolution of the variance differs between the models, can be used to measure the stochasticity of the radiation reaction. An increase in the variance of the energy distribution of electrons must be due to the probabilistic nature of the emission. As further work we propose a comparison of QED-PIC simulations of laser absorption in laser-plasma interactions using the different emission models and an investigation of the use of the variance to observe stochasticity in three-dimensional simulations of the interaction of a focusing laser pulse with a counter-propagating electron bunch produced by laser wakefield acceleration (with a realistic energy spectrum).

\section{Conclusions}

We have derived equations for the evolution of the expectation value of the momentum and variance in the energy of an electron population subject to three different radiation reaction models. We have considered classical and modified classical models, where the radiation reaction is deterministic and the power emitted is the classical synchrotron power in the former case and in the latter case accounts for reduction to the power emitted by quantum effects (the Gaunt factor $g$ ). We have also considered a stochastic model which calculates the emission using a more physically correct probabilistic treatment. We have shown that the expectation value of the energy evolves in almost the same way for the stochastic and modified classical models but differently for the classical model. The variance of the energy distribution evolves differently for all the models. This suggests that measuring the decrease in the expectation value of the energy is sufficient to measure the Gaunt factor but that a measurement of the variance is required to distinguish quantum stochastic effects.

\section{Acknowledgements}

This work was funded by Engineering and Physical Science Research Council grants EP/M018156/1, EP/M018091/1 and EP/M018555/1 and partially by the Knut 
and Alice Wallenberg Foundation (T.G.B., M.M.). Data access: the data required to reproduce the simulation results presented here are available at doi: 10.15124/ 332b52e8-7687-4c74-899b-eeclecb46875. The derivation of the analytical results presented here is given in appendix B.

\section{Appendix A. Functions describing synchrotron emission} is

The rate of photon emission (making the quasi-static and weak-field approximations)

$$
\lambda_{\gamma}(\eta)=\frac{\sqrt{3} \alpha_{f} c}{\lambda_{c}} \frac{\eta}{\gamma} h(\eta) \quad h(\eta)=\int_{0}^{\eta / 2} d \chi \frac{F(\eta, \chi)}{\chi} .
$$

The quantum synchrotron function is given in Sokolov \& Ternov (1968, equation (6.5)). In our notation it is, for $\chi<\eta / 2$,

$$
F(\eta, \chi)=\frac{4 \chi^{2}}{\eta^{2}} y K_{2 / 3}(y)+\left(1-\frac{2 \chi}{\eta}\right) y \int_{y}^{\infty} \mathrm{d} t K_{5 / 3}(t),
$$

where $y=4 \chi /[3 \eta(\eta-2 \chi)]$ and $\mathrm{K}_{n}$ are modified Bessel functions of the second kind. For $\chi \geqslant \eta / 2, F(\eta, \chi)=0$. In the classical limit $\hbar \rightarrow 0$ the quantum synchrotron spectrum reduces to the classical synchrotron spectrum $F(\eta, \chi) \rightarrow F_{\mathrm{cl}}\left(y_{c}\right)=y_{c}$ $\int_{y_{c}}^{\infty} \mathrm{d} u K_{5 / 3}(u) ; y_{c}=4 \chi / 3 \eta^{2}$. The probability that a photon is emitted with a given $\chi$ (by an electron with a given $\eta$ ) is $\rho_{\chi}(\eta, \chi) \mathrm{d} \chi=[1 / h(\eta)][F(\eta, \chi) / \chi] \mathrm{d} \chi$.

\section{Appendix B. Derivation of the moment equations}

We obtain an equation for the evolution of the expectation value of the electron momentum by multiplying (2.6) by $\boldsymbol{p}$ and integrating over momentum.

$$
n_{e}\left(\frac{\mathrm{d}\langle\boldsymbol{p}\rangle}{\mathrm{d} t}\right)_{s t}=-\int d^{3} \boldsymbol{p} \boldsymbol{p} \lambda_{\gamma}(\eta) f+\int d^{3} \boldsymbol{p} \boldsymbol{p} \frac{b}{2 m_{e} c} \int_{p}^{\infty} \mathrm{d} p^{\prime} \lambda_{\gamma}\left(\eta^{\prime}\right) \rho_{\chi}\left(\eta^{\prime}, \chi\right) \frac{p^{\prime 2}}{p^{2}} f\left(\boldsymbol{p}^{\prime}\right) .
$$

In spherical polars $d^{3} \boldsymbol{p}=p^{2} \mathrm{~d} p d^{2} \Omega$. We also write $\boldsymbol{p}=p \hat{\boldsymbol{p}}$. Therefore,

$$
n_{e}\left(\frac{\mathrm{d}\langle\boldsymbol{p}\rangle}{\mathrm{d} t}\right)_{s t}=-\int d^{3} \boldsymbol{p} \boldsymbol{p} \lambda_{\gamma}(\eta) f+\int d^{2} \Omega \frac{b \hat{\boldsymbol{p}}}{2 m_{e} c} \int_{0}^{\infty} \mathrm{d} p p \int_{p}^{\infty} \mathrm{d} p^{\prime} \lambda_{\gamma}\left(\eta^{\prime}\right) \rho_{\chi}\left(\eta^{\prime}, \chi\right) p^{\prime 2} f\left(\boldsymbol{p}^{\prime}\right) .
$$

We may exchange the order of integration over $p$ and $p^{\prime}$ in the second term on the right-hand side

$$
n_{e}\left(\frac{\mathrm{d}\langle\boldsymbol{p}\rangle}{\mathrm{d} t}\right)_{s t}=-\int d^{3} \boldsymbol{p} \boldsymbol{p} \lambda_{\gamma}(\eta) f+\int d^{2} \Omega \frac{b \hat{\boldsymbol{p}}}{2 m_{e} c} \int_{0}^{\infty} \mathrm{d} p^{\prime} \lambda_{\gamma}\left(\eta^{\prime}\right) f\left(\boldsymbol{p}^{\prime}\right) p^{\prime 2} \int_{0}^{p^{\prime}} \mathrm{d} p p \rho_{\chi}\left(\eta^{\prime}, \chi\right) .
$$

Here the $p$ dependence of $\rho_{\chi}$ is in $\chi=\left[\left(p^{\prime}-p\right) b\right] /\left(2 m_{e} c\right)$ (where we have assumed the electrons are ultra-relativistic). To simplify the identification of $g P_{\mathrm{cl}}$ we define $\rho_{h v} \mathrm{~d} h v$ as the probability that an electron with energy parameterised by $\eta$ emits a photon with energy $h v \cdot \rho_{\chi}=\rho_{h v}(\mathrm{~d} h v / \mathrm{d} \chi)=\rho_{h v}\left(2 m c^{2}\right) / b$. We may therefore write

$$
\begin{aligned}
n_{e}\left(\frac{\mathrm{d}\langle\boldsymbol{p}\rangle}{\mathrm{d} t}\right)_{s t}= & -\int d^{3} \boldsymbol{p} \boldsymbol{p} \lambda_{\gamma}(\eta) f+\int d^{2} \Omega \hat{\boldsymbol{p}} \int_{0}^{\infty} \mathrm{d} p^{\prime} \lambda_{\gamma}\left(\eta^{\prime}\right) f\left(\boldsymbol{p}^{\prime}\right) p^{\prime 2} \int_{0}^{p^{\prime} c} \mathrm{~d} h v \\
& \times\left(p^{\prime}-\frac{h v}{c}\right) \rho_{h v}\left(\eta^{\prime}, h v\right) .
\end{aligned}
$$


Now we use

$$
\int_{0}^{p^{\prime} c} \mathrm{~d} h v \rho_{h v}\left(\eta^{\prime}, h v\right)=1 \quad \int_{0}^{p^{\prime} c} \mathrm{~d} h v \rho_{h v}\left(\eta^{\prime}, h v\right) h v=(h v)_{\mathrm{av}}
$$

to get

$$
n_{e}\left(\frac{\mathrm{d}\langle\boldsymbol{p}\rangle}{\mathrm{d} t}\right)_{s t}=-\int d^{3} \boldsymbol{p} \boldsymbol{p} \lambda_{\gamma}(\eta) f+\int d^{3} \boldsymbol{p} \hat{\boldsymbol{p}} \lambda_{\gamma}(\eta) f(\boldsymbol{p})\left(p-\frac{(h v)_{\mathrm{av}}}{c}\right) .
$$

Cancelling the appropriate terms and identifying $g P_{\mathrm{cl}}=\lambda_{\gamma}(h v)_{\mathrm{av}}$ yields (3.2),

$$
\left(\frac{\mathrm{d}\langle\boldsymbol{p}\rangle}{\mathrm{d} t}\right)_{s t}=-\frac{\left\langle g P_{\mathrm{cl}} \hat{\boldsymbol{p}}\right\rangle}{c}
$$

The equation for the evolution of $\sigma^{2}$ (3.4) is obtained by using the same procedure to obtain an equation for $\left(\mathrm{d}\left\langle\gamma^{2}\right\rangle / \mathrm{d} t\right)_{s t}$, i.e. we multiply (2.6) by $\gamma^{2}$ and integrate over momentum,

$$
n_{e}\left(\frac{\mathrm{d}\left\langle\gamma^{2}\right\rangle}{\mathrm{d} t}\right)_{s t}=-\int d^{3} \boldsymbol{p} \gamma^{2} \lambda_{\gamma}(\eta) f+\int d^{3} \boldsymbol{p} \gamma^{2} \frac{b}{2 m_{e} c} \int_{p}^{\infty} \mathrm{d} p^{\prime} \lambda_{\gamma}\left(\eta^{\prime}\right) \rho_{\chi}\left(\eta^{\prime}, \chi\right) \frac{p^{\prime 2}}{p^{2}} f\left(\boldsymbol{p}^{\prime}\right),
$$

which can be written as

$$
\begin{aligned}
n_{e}\left(\frac{\mathrm{d}\left\langle\gamma^{2}\right\rangle}{\mathrm{d} t}\right)_{s t}= & -\int d^{3} \boldsymbol{p} \gamma^{2} \lambda_{\gamma}(\eta) f+\int d^{2} \Omega \int_{0}^{\infty} \mathrm{d} p^{\prime} \lambda_{\gamma}\left(\eta^{\prime}\right) f\left(\boldsymbol{p}^{\prime}\right) p^{\prime 2} \int_{0}^{p^{\prime} c} \mathrm{~d} h v \\
& \times\left(\gamma^{\prime}-\frac{h v}{m_{e} c^{2}}\right)^{2} \rho_{h v}\left(\eta^{\prime}, h v\right),
\end{aligned}
$$

where we have assumed $\gamma^{\prime}=p^{\prime} / m_{e} c$. Defining

$$
\int_{0}^{p^{\prime} c} \mathrm{~d} h v \rho_{h v}\left(\eta^{\prime}, h v\right)(h v)^{2}=\left[(h v)^{2}\right]_{\mathrm{av}}
$$

gives

$n_{e}\left(\frac{\mathrm{d}\left\langle\gamma^{2}\right\rangle}{\mathrm{d} t}\right)_{s t}=-\int d^{3} \boldsymbol{p} \gamma^{2} \lambda_{\gamma}(\eta) f+\int d^{3} \boldsymbol{p} \lambda_{\gamma}(\eta) f(\boldsymbol{p})\left(\gamma^{2}-2 \gamma \frac{(h v)_{\mathrm{av}}}{m_{e} c^{2}}+\frac{\left[(h \nu)^{2}\right]_{\mathrm{av}}}{m_{e}^{2} c^{4}}\right)$.

We again cancel the appropriate terms and this time identify $S=\lambda_{\gamma}\left[(h v)^{2}\right]_{\mathrm{av}}$ as well as $g P_{\mathrm{cl}}=\lambda_{\gamma}(h v)_{\mathrm{av}}$ to get

$$
\left(\frac{\mathrm{d}\left\langle\gamma^{2}\right\rangle}{\mathrm{d} t}\right)_{s t}=-2 \frac{\left\langle\gamma g P_{\mathrm{cl}}\right\rangle}{m_{e} c^{2}}+\frac{\langle S\rangle}{m_{e}^{2} c^{4}}
$$

To get an equation for $\left(\mathrm{d} \sigma^{2} / \mathrm{d} t\right)_{s t}$ we identify $\sigma^{2}=\left\langle\gamma^{2}\right\rangle-\langle\gamma\rangle^{2}$. Therefore,

$$
\left(\frac{\mathrm{d} \sigma^{2}}{\mathrm{~d} t}\right)_{s t}=\left(\frac{\mathrm{d}\left\langle\gamma^{2}\right\rangle}{\mathrm{d} t}\right)_{s t}-\left(\frac{\mathrm{d}\langle\gamma\rangle^{2}}{\mathrm{~d} t}\right)_{s t}=\left(\frac{\mathrm{d}\left\langle\gamma^{2}\right\rangle}{\mathrm{d} t}\right)_{s t}-2\langle\gamma\rangle\left(\frac{\mathrm{d}\langle\gamma\rangle}{\mathrm{d} t}\right)_{s t} .
$$

Substituting the results for $\left(\mathrm{d}\left\langle\gamma^{2}\right\rangle / \mathrm{d} t\right)_{s t}$ and $(\mathrm{d}\langle\gamma\rangle / \mathrm{d} t)_{s t}=\left\langle g P_{\mathrm{cl}}\right\rangle /\left(m_{e} c^{2}\right)$ (the latter is obtained by taking the dot product of (3.2) with $\hat{\boldsymbol{p}}$ and assuming $p=\gamma m_{e} c$ ) gives the 
result in (3.4):

$$
\left(\frac{\mathrm{d} \sigma^{2}}{\mathrm{~d} t}\right)_{s t}=-2 \frac{\left\langle\gamma g P_{\mathrm{cl}}\right\rangle}{m_{e} c^{2}}+\frac{\langle S\rangle}{m_{e}^{2} c^{4}}+2\langle\gamma\rangle \frac{\left\langle g P_{\mathrm{cl}}\right\rangle}{m_{e} c^{2}}=-2 \frac{\left\langle\Delta \gamma g P_{\mathrm{cl}}\right\rangle}{m_{e} c^{2}}+\frac{\langle S\rangle}{m_{e}^{2} c^{4}} .
$$

Here we have used $\Delta \gamma=\gamma-\langle\gamma\rangle$.

The moments of the classical and modified classical emission operators are straightforwardly obtained by integration by parts. To obtain (3.3) for $(\mathrm{d}\langle\boldsymbol{p}\rangle / \mathrm{d} t)_{\operatorname{modcl}}$ we multiply the emission operator $(\partial f / \partial t)_{\mathrm{em}}^{\mathrm{modcl}}$ in $(2.5)$ by $\boldsymbol{p}$ and integrate over momentum

$$
n_{e}\left(\frac{\mathrm{d}\langle\boldsymbol{p}\rangle}{\mathrm{d} t}\right)_{\operatorname{modcl}}=\int d^{3} \boldsymbol{p} \frac{\boldsymbol{p}}{p^{2}} \frac{\partial}{\partial p}\left(p^{2} g \frac{P_{\mathrm{cl}}}{c} f\right) .
$$

Substituting $d^{3} \boldsymbol{p}=p^{2} \mathrm{~d} p d^{2} \Omega$ and $\boldsymbol{p}=p \hat{\boldsymbol{p}}$ and integrating by parts yields

$$
\begin{aligned}
n_{e}\left(\frac{\mathrm{d}\langle\boldsymbol{p}\rangle}{\mathrm{d} t}\right)_{\bmod \mathrm{cl}} & =\int d^{2} \Omega \hat{\boldsymbol{p}}\left(\left[p^{3} g \frac{P_{\mathrm{cl}}}{c} f\right]_{0}^{\infty}-\int_{0}^{\infty} \mathrm{d} p p^{2} g \frac{P_{\mathrm{cl}}}{c} f\right) \\
& =-\int d^{2} \Omega \hat{\boldsymbol{p}} \int_{0}^{\infty} \mathrm{d} p p^{2} g \frac{P_{\mathrm{cl}}}{c} f .
\end{aligned}
$$

We have used the fact that $f \rightarrow 0$ as $p \rightarrow \infty$ (faster than $p^{5}$ diverges) to get the last result. We have now derived (3.3)

$$
\left(\frac{\mathrm{d}\langle\boldsymbol{p}\rangle}{\mathrm{d} t}\right)_{\mathrm{modcl}}=-\frac{1}{n_{e}} \int d^{3} \boldsymbol{p} g \frac{P_{\mathrm{cl}}}{c} \hat{\boldsymbol{p}} f=-\frac{\left\langle g P_{\mathrm{cl}} \hat{\boldsymbol{p}}\right\rangle}{c} .
$$

To derive (3.7) for $\left(\mathrm{d} \sigma^{2} / \mathrm{d} t\right)_{\operatorname{modcl}}$ we first multiply the emission operator $(\partial f / \partial t)_{\mathrm{em}}^{\operatorname{modcl}}$ in (2.5) by $\gamma^{2}$ and integrate over momentum

$$
n_{e}\left(\frac{\mathrm{d}\left\langle\gamma^{2}\right\rangle}{\mathrm{d} t}\right)_{\text {modcl }}=\int d^{3} \boldsymbol{p} \frac{\gamma^{2}}{p^{2}} \frac{\partial}{\partial p}\left(p^{2} g \frac{P_{\mathrm{cl}}}{c} f\right) .
$$

Substituting $d^{3} \boldsymbol{p}=p^{2} \mathrm{~d} p d^{2} \Omega, \gamma=p /\left(m_{e} c\right)$ and integrating by parts yields

$$
\begin{aligned}
n_{e}\left(\frac{\mathrm{d}\left\langle\gamma^{2}\right\rangle}{\mathrm{d} t}\right)_{\operatorname{modcl}} & =\int d^{2} \Omega\left(\left[\frac{p^{4}}{m_{e}^{2} c^{2}} g \frac{P_{\mathrm{cl}}}{c} f\right]_{0}^{\infty}-2 \int_{0}^{\infty} \mathrm{d} p \frac{p^{3}}{m_{e}^{2} c^{2}} g \frac{P_{\mathrm{cl}}}{c} f\right) \\
& =-\int d^{2} \Omega \hat{\boldsymbol{p}} \int_{0}^{\infty} \mathrm{d} p p^{2} \gamma g \frac{P_{\mathrm{cl}}}{m_{e} c^{2}} f
\end{aligned}
$$

Again, we have used the fact that $f \rightarrow 0$ as $p \rightarrow \infty$ (this time faster than $p^{6}$ diverges) to get the final result. We may write this more compactly as

$$
\left(\frac{\mathrm{d}\left\langle\gamma^{2}\right\rangle}{\mathrm{d} t}\right)_{\operatorname{modcl}}=-\frac{2}{n_{e}} \int d^{3} \boldsymbol{p} \gamma g \frac{P_{\mathrm{cl}}}{m_{e} c^{2}} \hat{\boldsymbol{p}} f=-2 \frac{\left\langle\gamma g P_{\mathrm{cl}}\right\rangle}{m_{e} c^{2}} .
$$

We get (3.7) by identifying $\sigma^{2}=\left\langle\gamma^{2}\right\rangle-\langle\gamma\rangle^{2}$ and $\Delta \gamma=\gamma-\langle\gamma\rangle$,

$$
\left(\frac{\mathrm{d} \sigma^{2}}{\mathrm{~d} t}\right)_{\operatorname{modcl}}=-2 \frac{\left\langle\gamma g P_{\mathrm{cl}}\right\rangle}{m_{e} c^{2}}+2\langle\gamma\rangle \frac{\left\langle g P_{\mathrm{cl}}\right\rangle}{m_{e} c^{2}}=-2 \frac{\left\langle\Delta \gamma g P_{\mathrm{cl}}\right\rangle}{m_{e} c^{2}} .
$$




\section{Appendix C. Derivation of $\xi$}

For simplicity in what follows we define $\tau_{S}$ and $\tau_{R}$ as

$$
S=\frac{m_{e}^{2} c^{4}}{\tau_{S}} \gamma^{4} \quad P_{\mathrm{cl}}=\frac{m_{e} c^{2}}{\tau_{R}} \gamma^{2} .
$$

Then we may write $\xi$ as

$$
\xi=\frac{\tau_{R}}{2 \tau_{S}} \frac{\left\langle\gamma^{4}\right\rangle}{\left\langle\Delta \gamma \gamma^{2}\right\rangle}
$$

where we have set $g_{2}=g=1$. We may evaluate the averages by substituting $f=\left[1 /\left(2 W \gamma_{0} m_{e} c\right)\right] \delta\left(p_{y}\right) \delta\left(p_{z}\right)$ for $\gamma_{0} m_{e} c(1-W)<p_{x}<\gamma_{0} m_{e} c(1+W)$.

$$
\begin{aligned}
\left\langle\gamma^{4}\right\rangle & =\frac{1}{2 W \gamma_{0} m_{e} c} \int_{\gamma_{0} m_{e} c(1-W)}^{\gamma_{0} m_{e} c(1+W)} \gamma^{4} \mathrm{~d} p_{x}=\frac{\gamma_{0}^{4}}{10 W}\left[(1+W)^{5}-(1-W)^{5}\right] \\
& =\frac{\gamma_{0}^{4}}{5 W}\left(10 W^{3}+5 W+W^{5}\right)
\end{aligned}
$$

and

$$
\begin{aligned}
\left\langle\Delta \gamma \gamma^{2}\right\rangle & =\frac{1}{2 W \gamma_{0} m_{e} c} \int_{\gamma_{0} m_{e} c(1-W)}^{\gamma_{0} m_{e} c(1+W)}\left(\gamma-\gamma_{0}\right) \gamma^{2} \mathrm{~d} p_{x} \\
& =\frac{\gamma_{0}^{3}}{24 W}\left[(1-W)^{3}(1+3 W)-(1+W)^{3}(1-3 W)\right]=\frac{2 \gamma_{0}^{3}}{3} W^{2} .
\end{aligned}
$$

Substituting these results into (C2) yields (4.2)

$$
\xi=\frac{33}{64 \sqrt{3}}\left(10+5 W^{-2}+W^{2}\right) \eta_{0} \approx\left(3.0+1.5 W^{-2}+0.3 W^{2}\right) \eta_{0},
$$

where we have used $\tau_{S} / \tau_{R}=(55 b) /(16 \sqrt{3})$ and $\eta_{0}=\gamma_{0} b$.

\section{REFERENCES}

Andersen, K. K., Esberg, J., Knudsen, H., Thomsen, H. D., UggerhøJ, U. I., Sona, P., Mangiarotti, A., Ketel, T. J., Dizdar, A. \& Ballestrero, S. 2012 Experimental investigations of synchrotron radiation at the onset of the quantum regime. Phys. Rev. D 86, 072001 .

Arber, T. D., Bennett, K., Brady, C. S., Lawrence-Douglas, A., Ramsay, M. G., Sircombe, N. J., Gillies, P., Evans, R. G., Schmitz, H., Bell, A. R. et al. 2015 Contemporary particle-in-cell approach to laser-plasma modelling. Plasma Phys. Control. Fusion 57, 113001.

BAlER, V. N. \& KATKOV, V. M. 1968 Quasiclassical theory of bremsstrahlung by relativistic particles. Sov. Phys. JETP 26, 807-813.

Baier, V. N., KatKov, V. M. \& Strakhovenko, V. M. 1991 Quasiclassical theory of radiation and pair creation in crystals at high energy. Rad. Eff. 527, 122-123.

BAshinov, A. V. \& KIM, A. V. 2013 On the electrodynamic model of ultra-relativistic laser-plasma interactions caused by radiation reaction effects. Phys. Plasmas 20 (11), 113111.

Bell, A. R. \& Kirk, J. G. 2008 Possibility of prolific pair production with high-power lasers. Phys. Rev. Lett. 101, 200403.

BLACKBURN, T. G. 2015 Measuring quantum radiation reaction in laser-electron-beam collisions. Plasma Phys. Control. Fusion 57 (7), 075012. 
Blackburn, T. G., Ridgers, C. P., Kirk, J. G. \& Bell, A. R. 2014 Quantum radiation reaction in laser-electron-beam collisions. Phys. Rev. Lett. 112, 015001.

Brady, C. S., Ridgers, C. P., Arber, T. D., Bell, A. R. \& Kirk, J. G. 2012 Laser absorption in relativistically underdense plasmas by synchrotron radiation. Phys. Rev. Lett. 109, 245006.

Bula, C., McDonald, K. T., Prebys, E. J., Bamber, C., Boege, S., Kotseroglou, T., Melissinos, A. C., Meyerhofer, D. D., RAgG, W., Burke, D. L. et al. 1996 Observation of nonlinear effects in compton scattering. Phys. Rev. Lett. 76, 3116-3119.

Bulanov, S. S., Chen, M., Schroeder, C. B., Esarey, E., Leemans, W. P., Bulanov, S. V., Esirkepov, T. Zh., KAndo, M., KogA, J. K., ZhidKov, A. G. et al. 2012 On the design of experiments to study extreme field limits high-quality electron beams from a laser wakefield accelerator using plasma-channel guiding. AIP Conf. Proc. 1507, 825.

Burke, D. L., Field, R. C., Horton-Smith, G., Spencer, J. E., Walz, D., Berridge, S. C., Bugg, W. M., Shmakov, K., Weidemann, A. W., Bula, C. et al. 1997 Positron production in multiphoton light-by-light scattering. Phys. Rev. Lett. 79, 1626-1629.

DiPinzzA, A. 2008 Exact solution of the Landau-Lifshitz equation in a plane wave. Lett. Math. Phys. 83, 305.

Di Piazza, A., Hatsagortsyan, K. Z. \& Keitel, C. H. 2010 Quantum radiation reaction effects in multiphoton compton scattering. Phys. Rev. Lett. 105, 220403.

Dinu, V., Harvey, C., Ilderton, A., Marklund, M. \& Torgrimsson, G. 2016 Quantum radiation reaction: from interference to incoherence. Phys. Rev. Lett. 116, 044801.

Duclous, R., KirK, J. G. \& Bell, A. R. 2011 Energy straggling and radiation reaction for magnetic bremsstrahlung. Plasma Phys. Control. Fusion 53, 015009.

Elkina, N. V., Fedotov, A. M., Kostyukov, I. Y., Legkov, M. V., Narozhny, N. B., Nerush, E. N. \& RUHL, H. 2011 Qed cascades induced by circularly polarized laser fields. Phys. Rev. ST Accel. Beams 14, 054401.

ERBER, T. 1966 High-energy electromagnetic conversion processes in intense magnetic fields. Rev. Mod. Phys. 38, 626-659.

Faure, J., Glinec, Y., Pukhov, A., Kiselev, S., Gordienko, S., Lefebvre, E., Rousseau, J. P., Burgy, F. \& MalkA, V. 2004 A laser-plasma accelerator producing monoenergetic electron beams. Nature 431, 541-544.

FURrY, W. H. 1951 On bound states and scattering in positron theory. Phys. Rev. 81, 115-124.

Geddes, C. G. R., Toth, Cs., van Tilborg, J., Esarey, E., Schroeder, C. B., Bruhwiler, D., Nieter, C., Cary, J. \& Leemans, W. P. 2004 High-quality electron beams from a laser wakefield accelerator using plasma-channel guiding. Nature 431, 538-541.

Gonoskov, A., Bastrakov, S., Efimenko, E., Ilderton, A., Marklund, M., Meyerov, I., Muraviev, A., Sergeev, A., Surmin, I. \& Wallin, E. 2015 Extended particle-in-cell schemes for physics in ultrastrong laser fields: review and developments. Phys. Rev. E 92, 023305 .

Harvey, C. N., Gonoskov, A., Ilderton, A. \& Marklund, M. 2017 Quantum quenching of radiation losses in short laser pulses. Phys. Rev. Lett. 118, 105004.

HeisenberG, W. \& Euler, H. 1936 Consequences of dirac theory of the positron. Z. Phys. 98, 714.

Ilderton, A. \& Torgrimsson, G. 2013 Radiation reaction in strong-field qed. Phys. Lett. B 725, 481.

KIRK, J. G., Bell, A. R. \& ARKA, I. 2009 Pair production in counter-propagating laser beams. Plasma Phys. Control. Fusion 52, 085008.

KRiVitskiI, V. \& TSYTovich, V. 1991 Average radiation-reaction force in quantum electrodynamics. Sov. Phys. Uspekhi 34 (3), 250-258; cited by 28.

Landau, L. D. \& Lifshitz, E. M. 1987 The Classical Theory of Fields, The Course of Theoretical Physics, vol. 2. Butterworth-Heinemann.

Leemans, W. P., Gonsalves, A. J., Mao, H.-S., Nakamura, K., Benedetti, C., Schroeder, C. B., Tóth, C., Daniels, J., Mittelberger, D. E., Bulanov, S. S. et al. 2014 Multi$\mathrm{GeV}$ electron beams from capillary-discharge-guided subpetawatt laser pulses in the self-trapping regime. Phys. Rev. Lett. 113, 245002. 
Mangles, S., Murphy, C. D., Najmudin, Z., Thomas, A. G. R., Collier, J. L., Dangor, A. E., Divall, E. J., Foster, P. S., Gallacher, J. G., Hooker, C. J. et al. 2004 Monoenergetic beams of relativistic electrons from intense laser-plasma interactions. Nature 431, 535-538.

Neitz, N. \& Di PiazzA, A. 2013 Stochasticity effects in quantum radiation reaction. Phys. Rev. Lett. 111, 054802.

Ridgers, C. P., Kirk, J. G., Duclous, R., Blackburn, T. G., Brady, C. S., Bennett, K., ARber, T. D. \& Bell, A. R. 2014 Pair production in counter-propagating laser beams. J. Comput. Phys. 260, 273.

Ritus, V. I. 1985 Quantum effects of the interaction of elementary particles with an intense electromagnetic field. J. Russ. Laser Res. 6, 497.

Sarri, G., Corvan, D. J., Schumaker, W., Cole, J. M., Di Piazza, A., Ahmed, H., Harvey, C., Keitel, C. H., Krushelnick, K., Mangles, S. P. D. et al. 2014 Ultrahigh brilliance multi-mev $\gamma$-ray beams from nonlinear relativistic thomson scattering. Phys. Rev. Lett. 113, 224801.

SHEN, C. S. \& White, D. 1972 Energy straggling and radiation reaction for magnetic bremsstrahlung. Phys. Rev. Lett. 28, 455.

Sokolov, A. A. \& Ternov, I. M. 1968 Synchrotron Radiation. Akademie-Verlag.

Sokolov, I. V., Naumova, N. M., Nees, J. A. \& Mourou, G. A. 2010 Pair creation in qed-strong pulsed laser fields interacting with electron beams. Phys. Rev. Lett. 105, 195005.

Tajima, T. \& Dawson, J. M. 1979 Laser electron accelerator. Phys. Rev. Lett. 43, 267-270.

Thomas, A. G. R., Ridgers, C. P., Bulanov, S. S., Griffin, B. J. \& Mangles, S. P. D. 2012 Strong radiation-damping effects in a gamma-ray source generated by the interaction of a high-intensity laser with a wakefield-accelerated electron beam. Phys. Rev. X 2, 041004.

Vranic, M., Grismayer, T., Fonseca, R. A. \& Silva, L. O. 2015 Quantum radiation reaction in head-on laser-electron beam interaction. New J. Phys. 18, 073035.

Vranic, M., Martins, J. L., Vieira, J., Fonseca, R. A. \& Silva, L. O. 2014 All-optical radiation reaction at $10^{21} \mathrm{~W} \mathrm{~cm}^{-2}$. Phys. Rev. Lett. 113, 134801.

WANG, H. Y., YAN, X. Q. \& ZEPF, M. 2015 Signatures of quantum radiation reaction in laserelectron-beam collisions. Phys. Plasmas 22 (9), 093103.

Zhang, P., Ridgers, C. P. \& Thomas, A. G. R. 2015 The effect of nonlinear quantum electrodynamics on relativistic transparency and laser absorption in ultra-relativistic plasmas. New J. Phys. 27, 043051. 